\title{
Research Paper \\ Research Databases and Geriatrics and Gerontology Journals: The Comparison of Data- bases and Their Covered Journals
}

\author{
*Mansoureh Feizabadi ${ }^{1,2}$, Maryam Nakhoda ${ }^{1}$, Ahmad Delbari ${ }^{3}$
}

1. Department of Information Science and Knowledge Studies, Faculty of Management, University of Tehran, Tehran, Iran.

2. Deputy of Research and Technology, Sabzevar University of Medical Sciences, Sabzevar, Iran.

3. Iranian Research Center on Aging, University of Social Welfare and Rehabilitation, Tehran, Iran.

Received: 28 Mar. 2016 Accepted: 20 Jun. 2016

Key words:

Geriatrics, Journals, Databases as topic, Traditional overlapping

\section{ABSTRACT}

Objectives The database and the journals indexed under them are the most important information resources for students and experts in various disciplines, including geriatrics. Given the multiplicity of databases and high subscription fees of the journals, increased awareness regarding those databases covering the majority of journals related to the topic of geriatrics is a necessity for the libraries, experts, and students in this field.

Methods \& Materials Quantitative content analysis and applied research were used in this study. The statistical population consisted of 177 active scientific electronic journals that were extracted by searching the keyword 'Geriatrics' through the Ulrichs web. The SPSS 19 software and Excel were used for data analysis. Descriptive- statistics and traditional overlap percentage of 14 databases and indexing services were also studied.

Results The results showed that the PubMed database covering 109 journals (61.58\%) had the largest coverage of journals in the field of Geriatrics and Gerontology. Among the noted journal publishers, Elsevier was at the top rank with 15 Geriatrics and Gerontology journals (8.5\%) indexed on PubMed. The largest providers of full-text articles was EBSCO host covering 104 journals (58.75\%). The place holder for the highest rate of journals covered under database overlapped with the two databases namely Current Contents and the Web of Science with both of them covering $82.09 \%$ of the journals.

Conclusion The findings of the research indicated that the search engines and suppliers of information for users such as libraries could compare the high rates of overlapping with each other in order to subscribe the databases and reduce costs.

\section{* Corresponding Author:}

Mansoureh Feizabadi, PhD Candidate

Address: Department of Information Science and Knowledge Studies, Faculty of Management, University of Tehran, Tehran, Iran.

Tel: +98 (21) 88003575

E-mail: feizabadi_mns@ut.ac.ir 


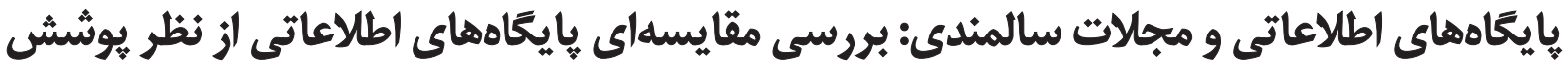

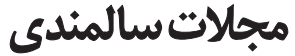

\author{
"منصوره فيض آبادى “'، مريم ناخدا'، احمد دلبرى" \\ 1- كروه علوم اطلاعات و دانششناسى، دانشكده مديريت، دانشكاه تهران، تهران، ايران.

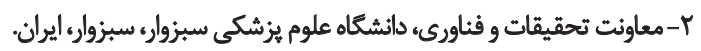

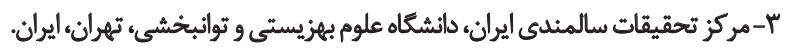

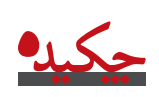

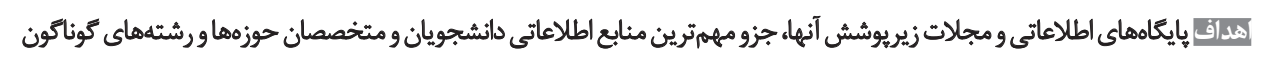

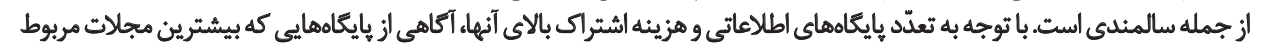

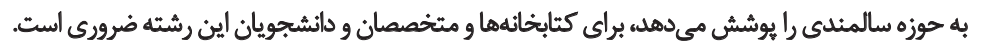

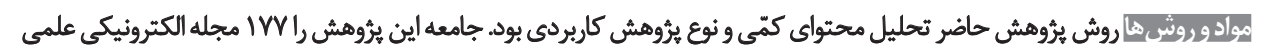

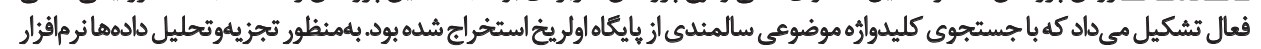

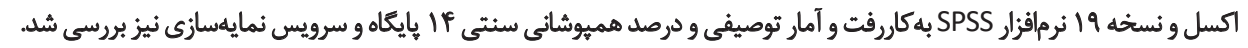

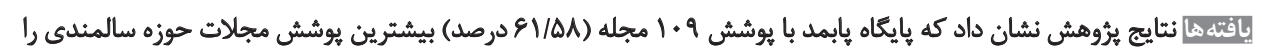

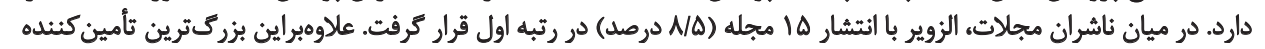

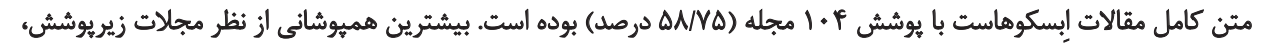

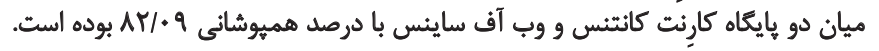

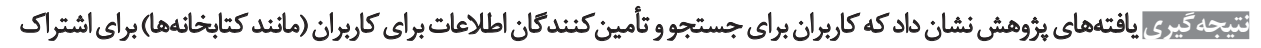

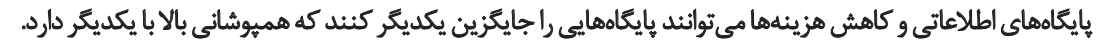

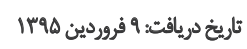

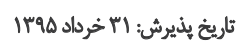

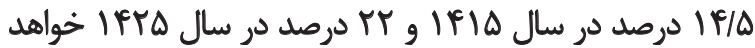

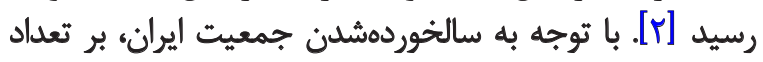

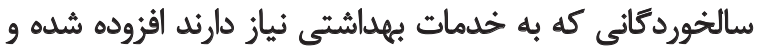

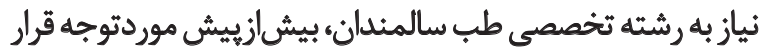

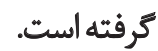

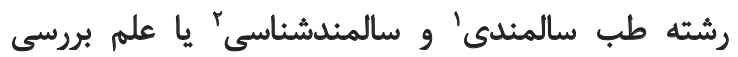

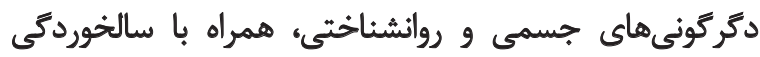

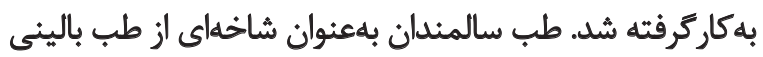

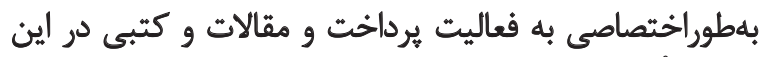

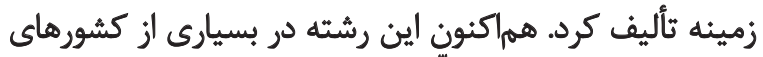

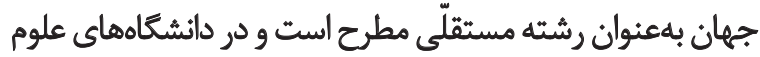

1. Geriatrics

2. Gerontology
مقدمه

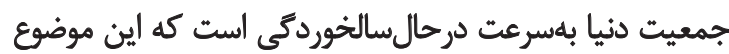

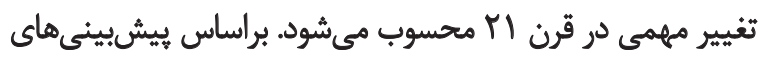

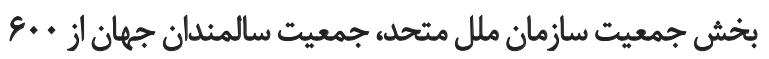

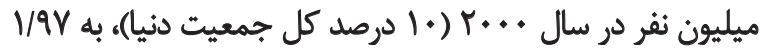

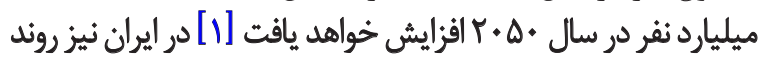

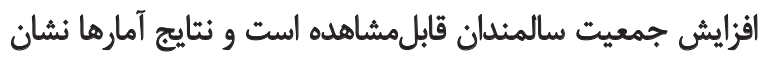

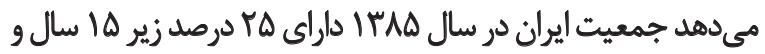

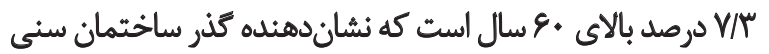

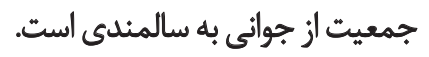

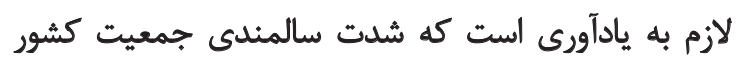

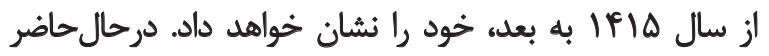

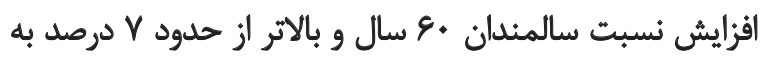


تهيه منابع مشابه جلوكيرى مى كند [9]]

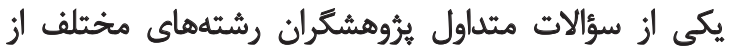

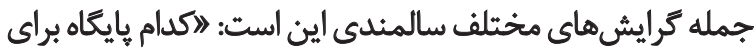

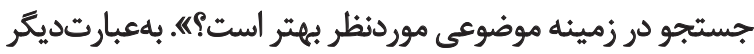

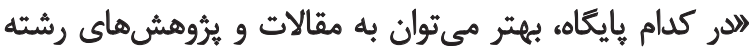

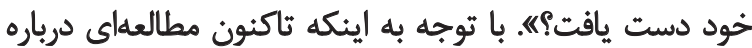

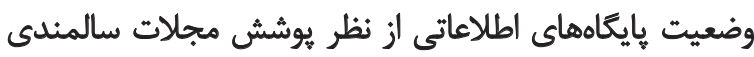

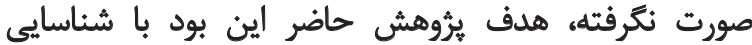

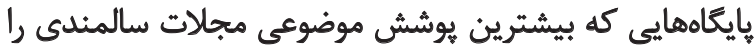

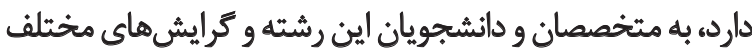

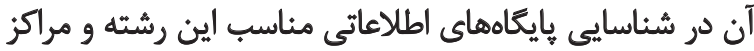

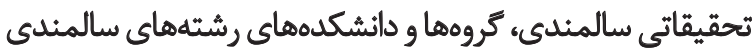

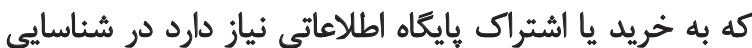
بإيكاه اطلاعاتي مناسب كمك كند.

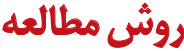

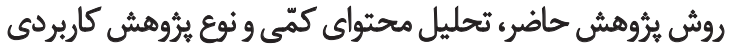

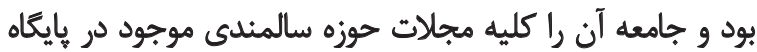

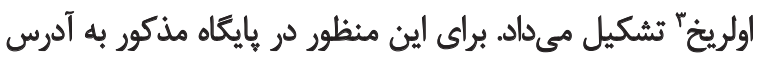
بttps://ulrichsweb-serialssolutions-com با استفاده از كليدوازثه موضوعى سالمندى جستجو شد. جامعترين

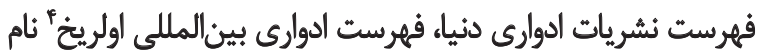
دارد كه اصلىترين ابزار براى شناسايى و انتخاب مجلات مونات موردئياز

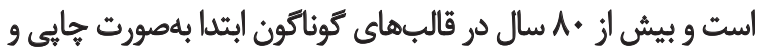

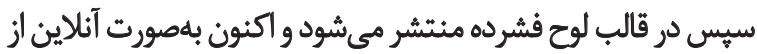

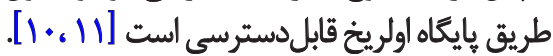

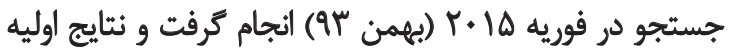

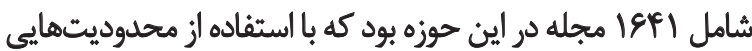

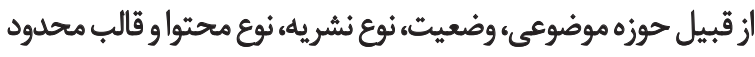

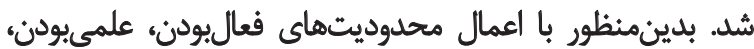

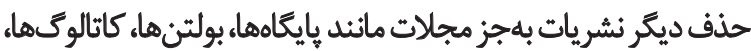

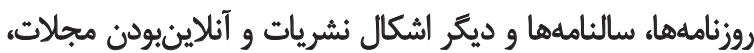

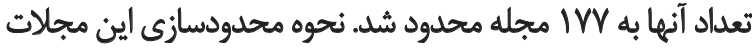
در تصوير شماره ا مشخص شده است. يس إز تعيين مجلات موردنظر، از نتايج جستجو در بايكاه خروجي

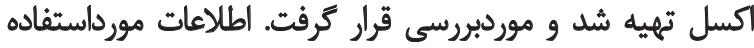

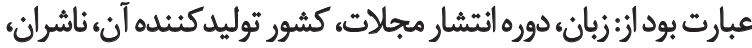

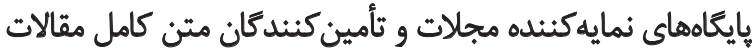

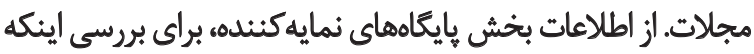

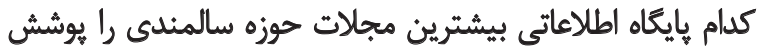

3. Ulrich web

4. Ulrich's international periodicals directory
يزشكىى، متخصصان اين رشته راتربيت مي كند ["]].

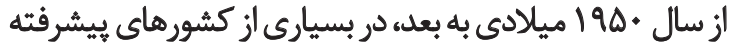

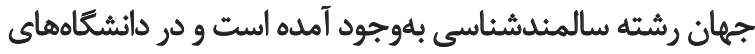

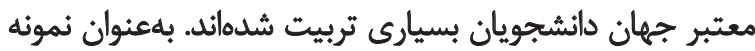

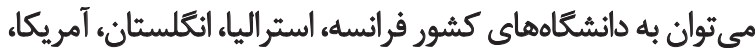

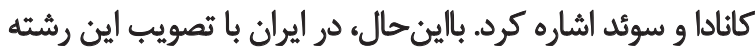

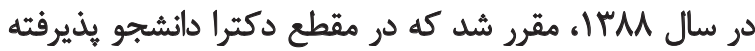

$$
\text { شود. }
$$

امروزه علاوهبر اين دو تخصص، ديكر كرايشهاي مربوط به به درئ

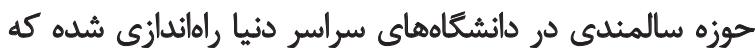

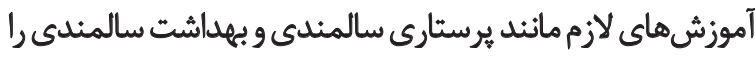

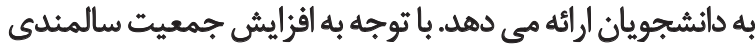

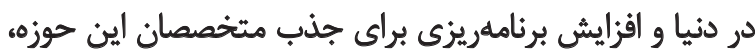

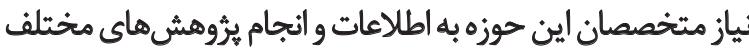

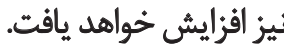

يايكاههاى اطلاعاتى ومجلات مرتبط، از جمله مهرمترين مكانها

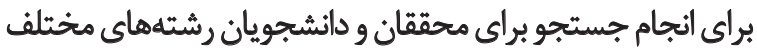

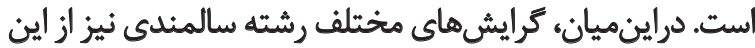

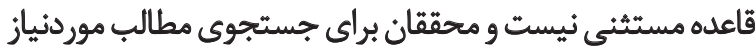

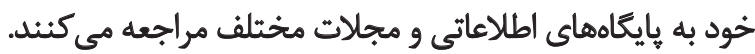

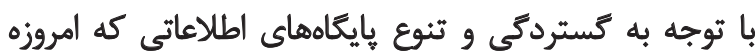

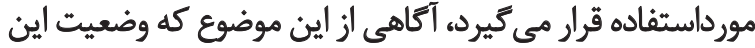

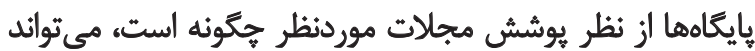

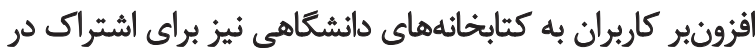

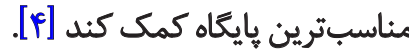
با توجه به اينكه اين بايخامها هزينه هئكفتى دارد و در عناوين

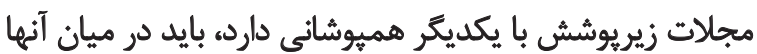

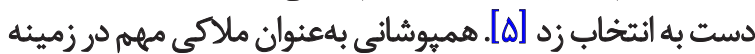

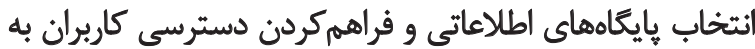

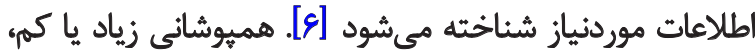

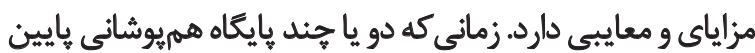

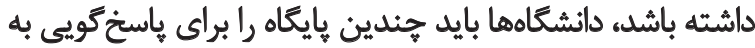

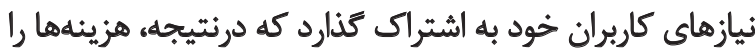

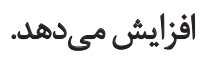

ازيكسو، كاربر براي يافتن اطلاعات مناسب خود بايد در

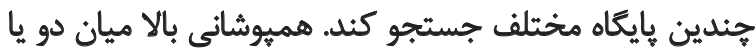

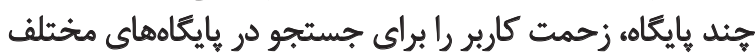

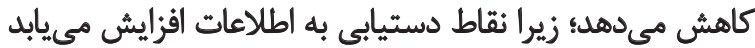

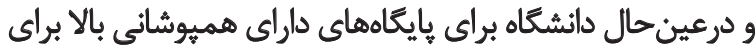

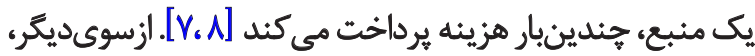

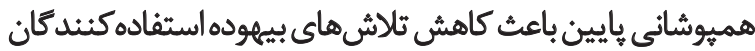

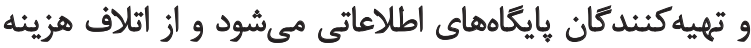



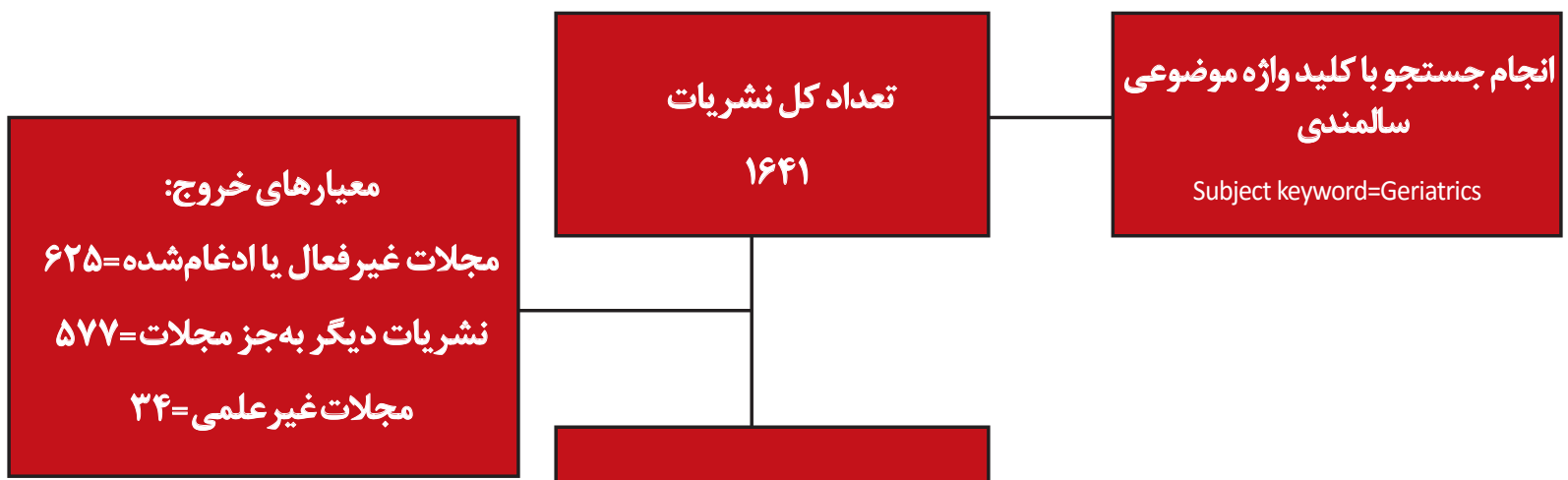

تصوير ا. فرايند جستجو و محدودسازى نشريات موردبررسى در بايكاه اولريخ.

در بايكاه اولريخ نمايه ميشده انجام كرفت. نتايج يُروهش نشان

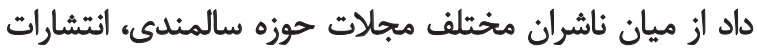

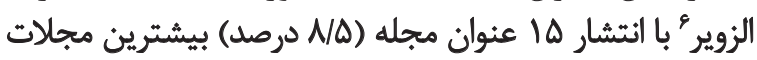

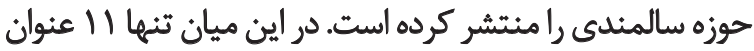

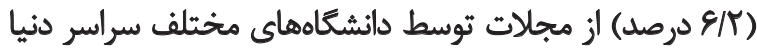

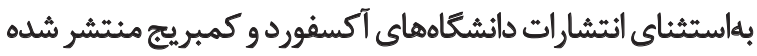

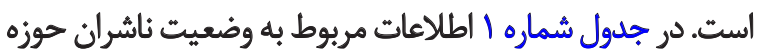

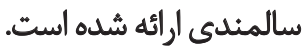

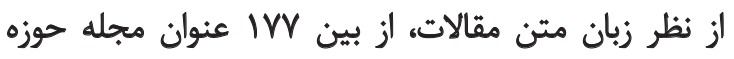

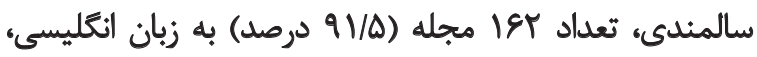

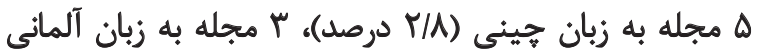

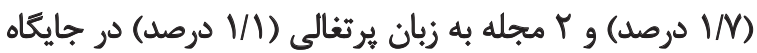

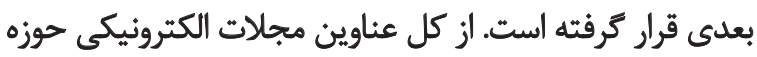

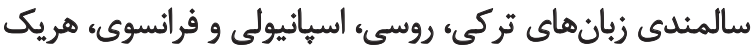

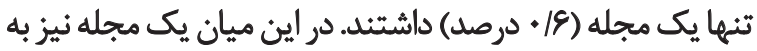

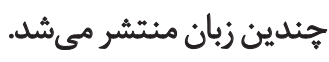

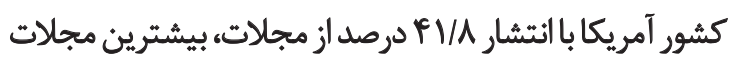

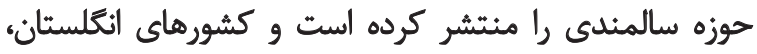

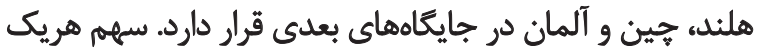

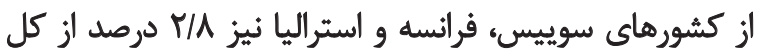

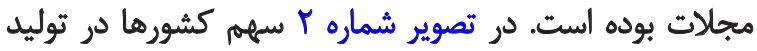
مجلات حوزه سالمندى ارائه شده است.
مي دهد، استفاده شد.

براى بررسى ميزان هميوشانى هايكاهها از نظر تعداد مجلات

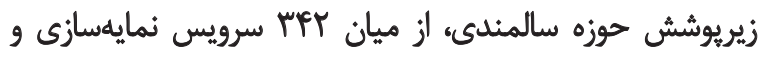

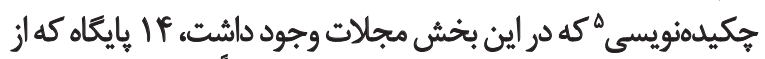

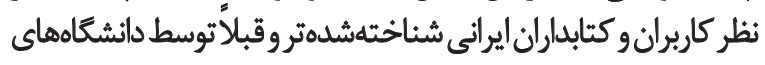

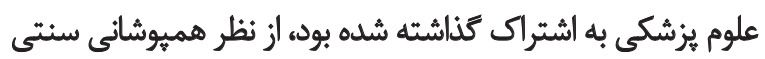

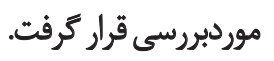

براى بررسى هميوشانى مجلات در ميان خايكاهها، از هميوشانى

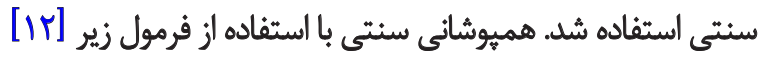
و نسبت اشتراك و اجتماع نشريات نمايهسازي شانده توسط دو بيايكًاه

$$
\text { محاسبه مي شود. }
$$

AnB=فقط فهرست نشرياتى كه همزمان توسط دو سرويس نمايهسازى يا بايكًاه نمايه شدهاند.

نمايرازي تهرست همه نُشريات نمايهشده توسط يك يا هر دوخدمت AUB نمايهسازى [1 [1 ].

batọ يروهش حاضر روى IVV مجله آنلاين حوزه سالمندى كه $\%$ Overlap $=\frac{n(A \cap B)}{n(A \cup B)} \times 100$

5. Abstracting/Indexing 
جدول ا. فراوائى و درصد ناشران مجلات حوزه سالمندى.

\begin{tabular}{|c|c|c|}
\hline دوصد & تعداد & 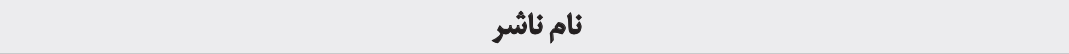 \\
\hline Na & 10 & Elsevier \\
\hline$V / q$ & if & Routledge \\
\hline $8 / 1$ & ir & Springer \\
\hline $9 / \pi$ & 11 & Universities \\
\hline$\Delta / 8$ & 1. & Sage Publications \\
\hline$\Delta / 1$ & 9 & Associations \& Scientific Communities \\
\hline$r / F$ & $\&$ & Wiley-Blackwell Publishing \\
\hline$r / r$ & f & BioMed Central, Cambridge University Press, Oxford University Press, Institutes \\
\hline $\mathbb{I N}$ & r & S. Karger, Lippincott Williams \& Wilkins \\
\hline $1 /$ & r & Bentham Open, W.B. Saunders Co, Taylor \& Francis \\
\hline+18 & 1 & John Wiley \& Sons, Excerpta Medica, McGraw-Hill \\
\hline "q & eq & ن ناشران ديكر \\
\hline 1.. & in & 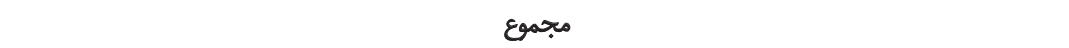 \\
\hline
\end{tabular}

乩

كرفته است. در اين ميان 9/1 درصد از مجلات، وضعيت فاصله

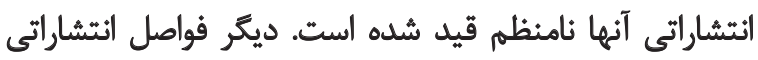

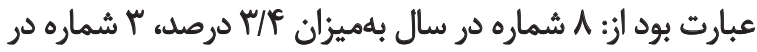

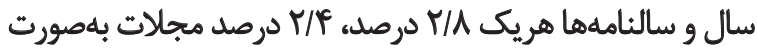

از نظر فاصله و نوبت انتشار، يافتهها نشان داد تعداد وV مجله

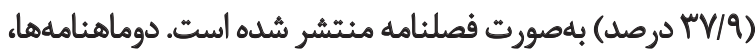

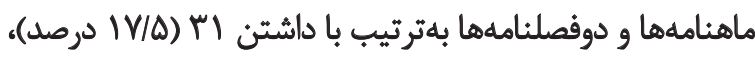

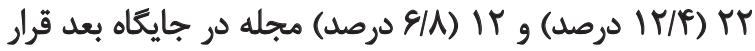

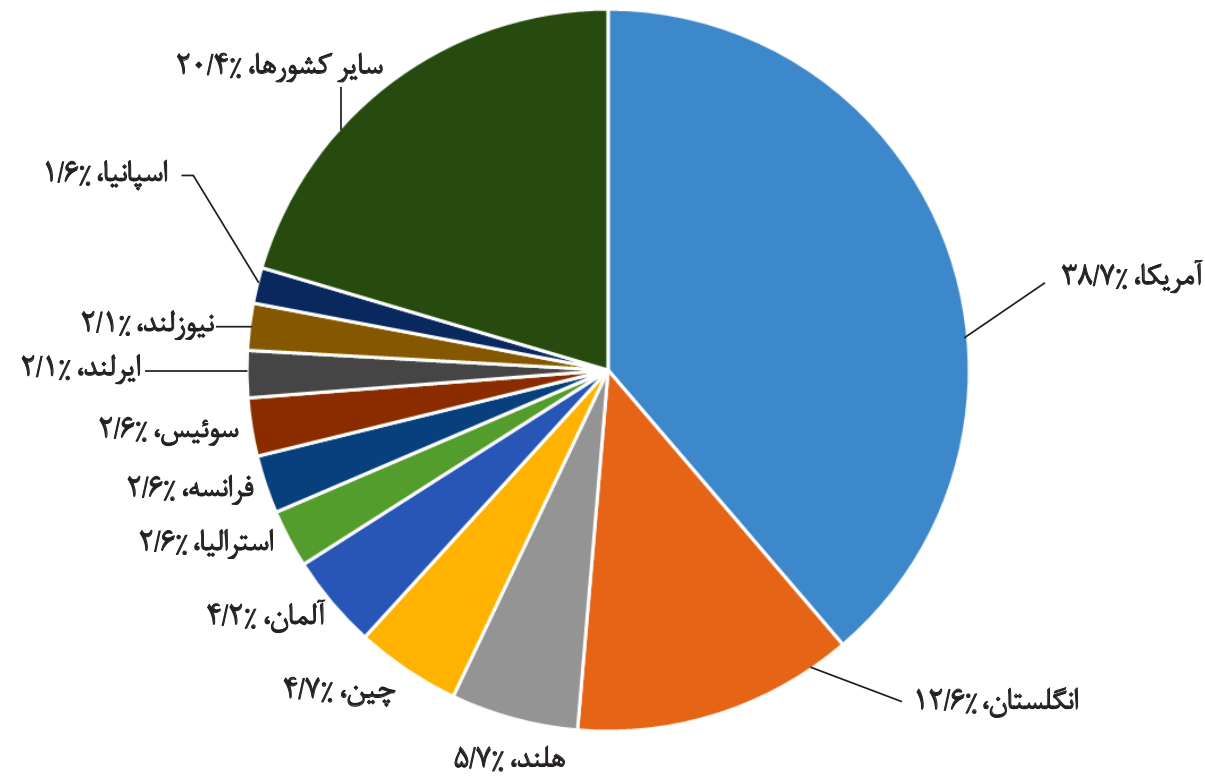


جدول r. بايكّاههاي نمايهكنده مجلات حوزه سالمندى

\begin{tabular}{|c|c|c|}
\hline درصد & تعداد & ثام هايخاه \\
\hline $8 / / 01$ & 1.9 & PubMed \\
\hline $81 / .1$ & 1.1 & Scopus \\
\hline FNAA & $N$ & EMBASE \\
\hline WV/TA & \& & Web of Science \\
\hline$M \Psi / M$ & $\Delta q$ & MEDLINE \\
\hline rI/gr & $\Delta$ & Current Contents \\
\hline Yq/qY & $\Delta$ & PsycINFO \\
\hline$r q / T r$ & Qr & Excerpta Medica \\
\hline$r q / T$ & ar & PsycFIRST \\
\hline TNMP & ه. & Science Citation Index Expanded \\
\hline$r v / I$ & in & AgeLine \\
\hline ro/PT & PA & CINAHL \\
\hline$W \cdot V$ & m & Social Sciences Citation Index \\
\hline $\mid N / \Delta \Delta$ & M & CAB Abstracts \\
\hline $\mid W / \Delta \Delta$ & $\pi f$ & Global Health \\
\hline $1 \cdot / \mathrm{N}^{w}$ & 19 & Chemical Abstracts \\
\hline Ner & 10 & Biological Abstracts \\
\hline
\end{tabular}

L

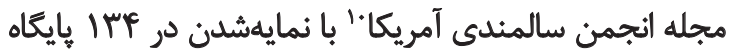

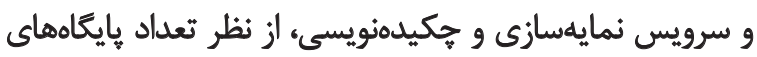

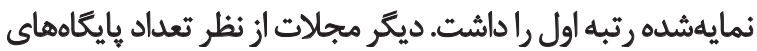

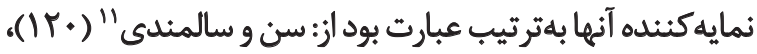

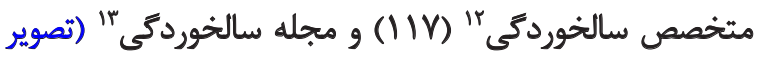
شماره (r).

در مورد وضعيت هميوشاني و تعلياد مجلات مشترك ميان

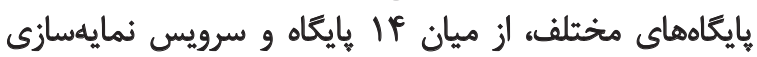
و خكيدهنويسى مجلات الكترونيكى حوزه سالمندى، يايكاه

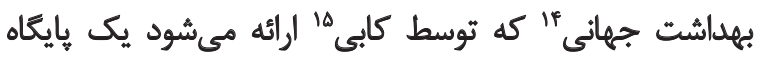

10. American geriatrics society journal

11. Age and ageing

12. The gerontologist

13. Journals of gerontology

14. Global health web

15. $\mathrm{CABI}$
ه شماره و • أشماره در سال منتشر شده است و ديكر فواصل

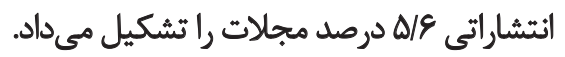

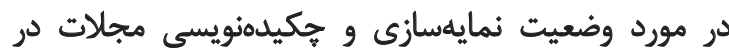

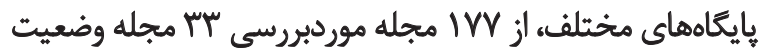

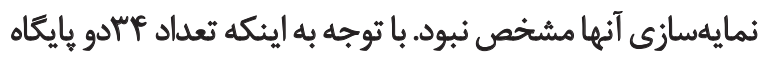

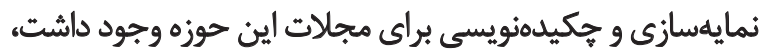

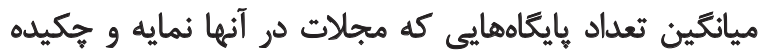

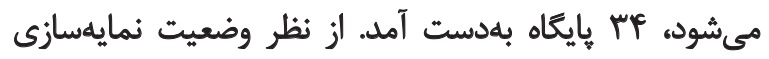

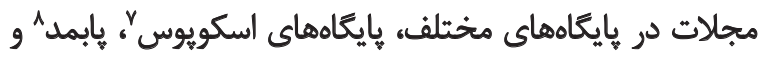

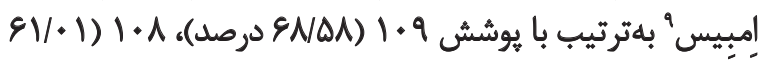

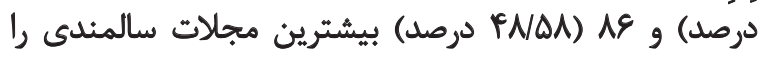

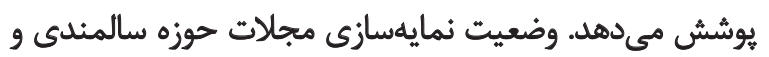

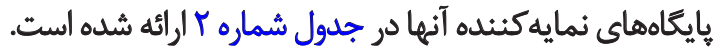
7. Scopus
8. PubMed
9. EMBASE 


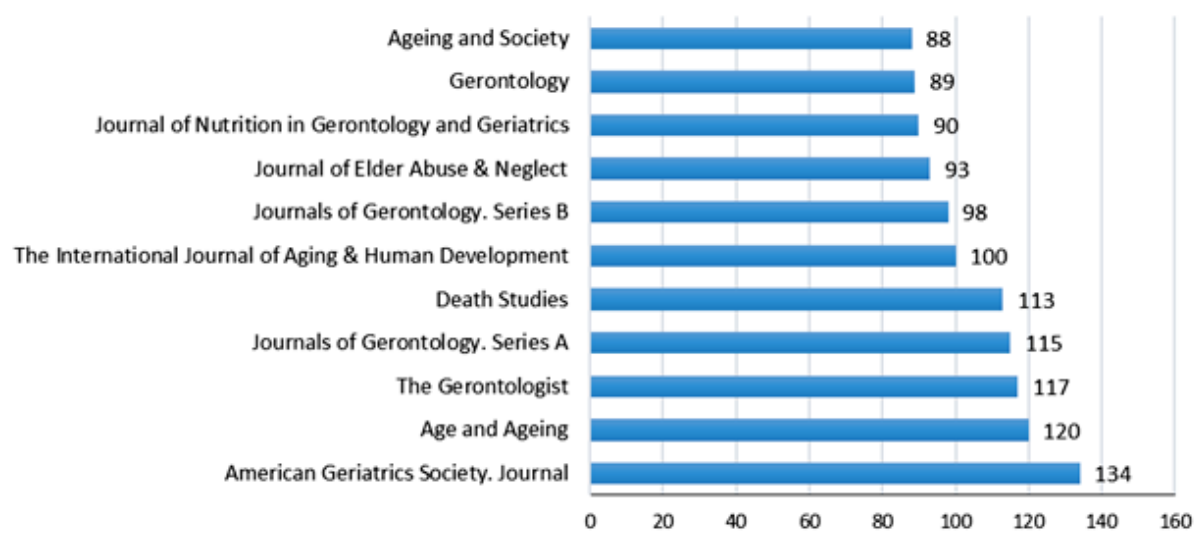

كتابشناختى اختصاصى در حوزه بهداشت عمومى است كه در د نشان داده شده است.

نتايج بروهش حاضر در مورد ميزان هميوشانى سنتى

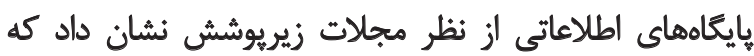
بالاترين درصد هميوشانى مجلات الكترونيكى حوزه سالمندى نائي

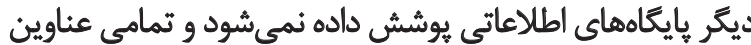

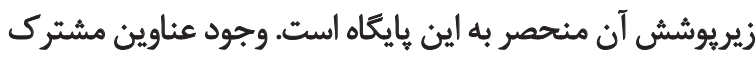

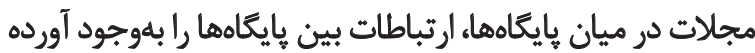

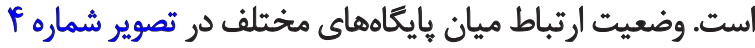

جدول ب. تعداد مجلات مشترك ميان خايكاهها و سرويسهاي نمايهسازى مجلات حوزه سالمئدى.

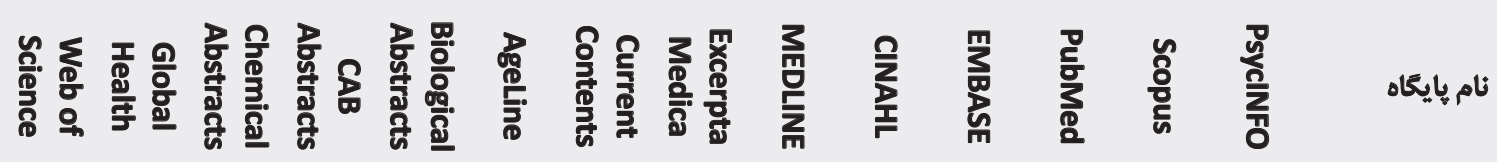

\begin{tabular}{|c|c|c|c|c|c|c|c|c|c|c|c|c|c|c|}
\hline MV & • & ir & $1 r$ & $\Lambda$ & mf & me & r. & pq & rq & Pr & ar & $\Delta r$ & + & PsycINFO \\
\hline gr & + & 19 & rI & if & iv & $\Delta \Delta$ & $p q$ & $\Delta A$ & fo & (1) & $q$ & + & $\Delta t$ & Scopus \\
\hline op & - & 19 & $M$ & 10 & er & $\Delta \Delta$ & in & $\Delta$ & erp & A. & - & $q$ & $\Delta r$ & PubMed \\
\hline$\Delta V$ & - & IA & 19 & if & $r \Delta$ & pq & $\Delta r$ & $\mathbb{A}$ & me & . & A. & A) & $m$ & EMBASE \\
\hline ri & - & 1. & 11 & $\Delta$ & m & $r$ & 10 & I & . & $r e$ & m & ro & $p q$ & CINAHL \\
\hline$p q$ & . & 18 & iv & 11 & $M$ & if & r & - & $M$ & $\mathbb{M}$ & $\Delta M$ & $\Delta$ & pq & MEDLINE \\
\hline$m p$ & - & 11 & Ir & 11 & ir & MV & . & rV & 10 & or & fA & $p q$ & r. & Excerpta Medica \\
\hline$\Delta \Delta$ & . & if & 18 & 1. & ri) & . & 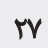 & pe & M & $p q$ & $\Delta \Delta$ & $\Delta \Delta$ & $m$ & Current Contents \\
\hline m & . & 9 & 1. & $\Delta$ & . & H & ir & Mr & $m$ & ro & Fr & pr & $m$ & Ageline \\
\hline ir & • & 1 & $\Lambda$ & - & $\Delta$ & $1+$ & 11 & 11 & $\Delta$ & if & 10 & if & $\wedge$ & Biological Abstracts \\
\hline IV & • & 16 & • & $\Lambda$ & 1 & 18 & it & IV & 11 & 19 & r & H & ir & CAB Abstracts \\
\hline 18 & • & + & ir & 1 & 9 & 18 & 11 & 18 & 1. & $M$ & 19 & 19 & ir & Chemical Abstracts \\
\hline - & - & . & . & . & . & . & . & . & . & . & . & . & - & Global Health \\
\hline . & . & 18 & IV & $1{ }^{H}$ & $m$ & $\Delta \Delta$ & me & $p q$ & $M$ & $\Delta V$ & of & Pr & $M$ & Web of Science \\
\hline
\end{tabular}

次 


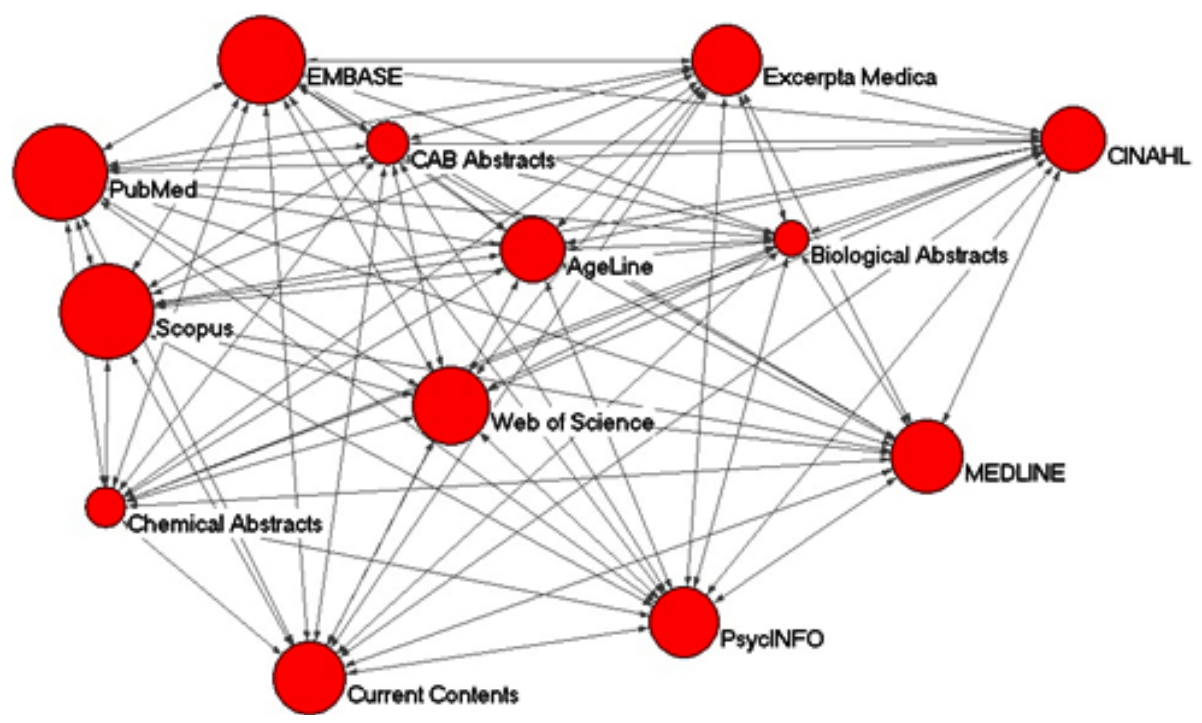

كرفت. نتايج يروهش نشان داد بيشترين مجلات، مربوط به كشور

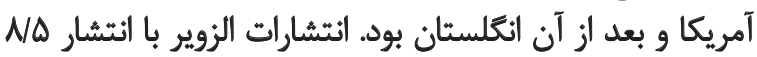

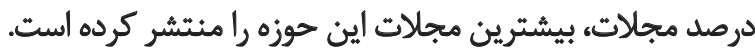

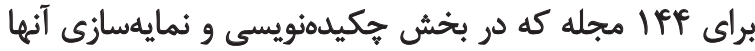

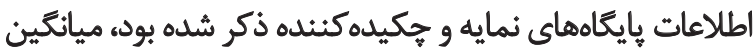

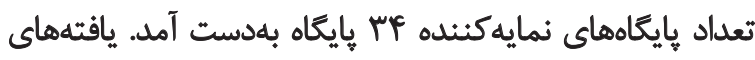

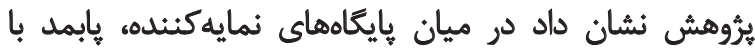

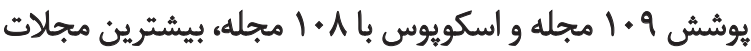

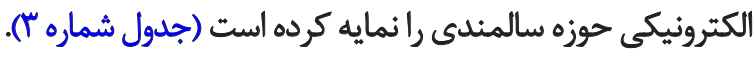
نتايج حاصل از بررسى هميوشانى سنتى ثايكاهها از نظر

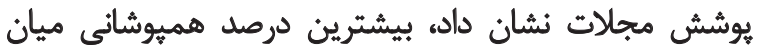

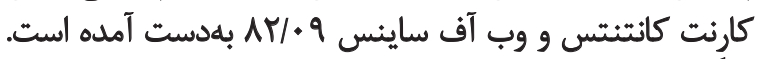

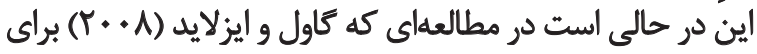

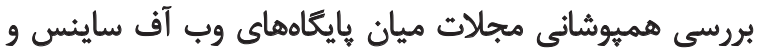

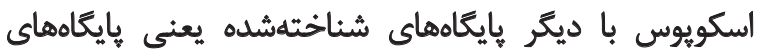

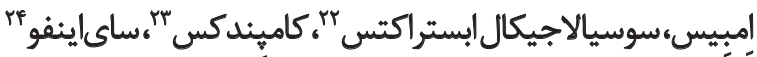

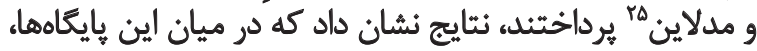

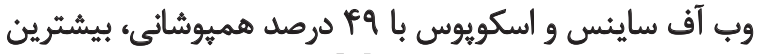

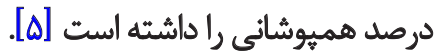

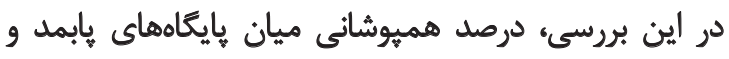

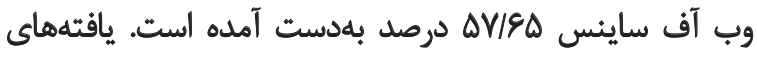

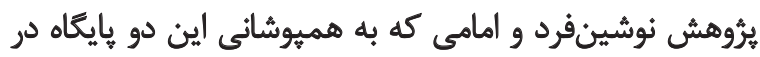

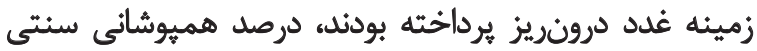

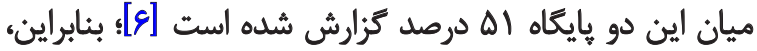

22. Sociological Abstracts

23. Compendex

24. PsycINFO

25. MedLine

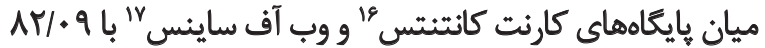

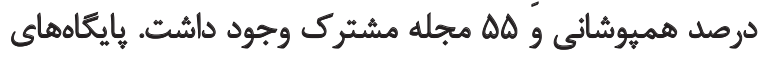

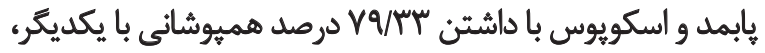

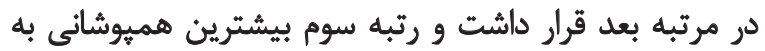

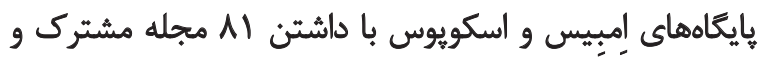

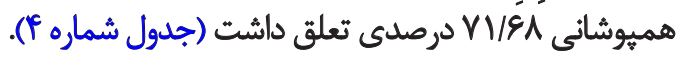
بهجز وايكاه بهداشت جهانى كه با هيجيك از بايكاهها عنوان

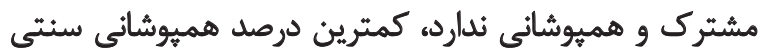

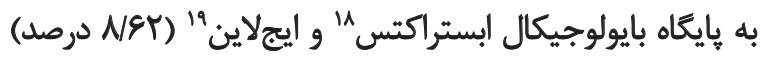

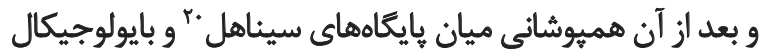
ابستراكتس (9 •/9 درصد) تعلق داشت ميت.

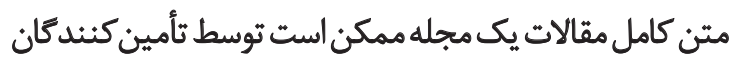

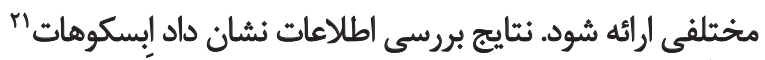

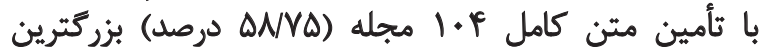

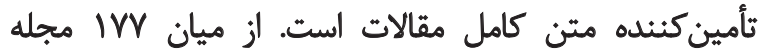

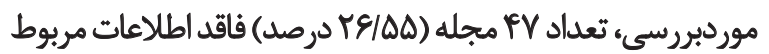

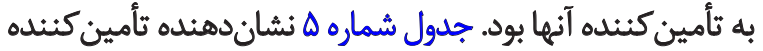

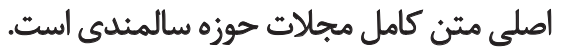
ثب در اين مطالعه، كليه مجلات الكترونيكى فعال در حوزه سالمندى كه در يايڤًاه اولريخ وجود داشت، موردمطالعه قرار

16. Current Contents

17. Web of Science

18. Biological Abstracts

19. AgeLine

20. CINAHL

21. EBSCOhost 
جدول P. درصد هميوشائى سنتي ميان بائكامهاى اطلاعائي نمايه كننده مجلات حوزه سالمندى.

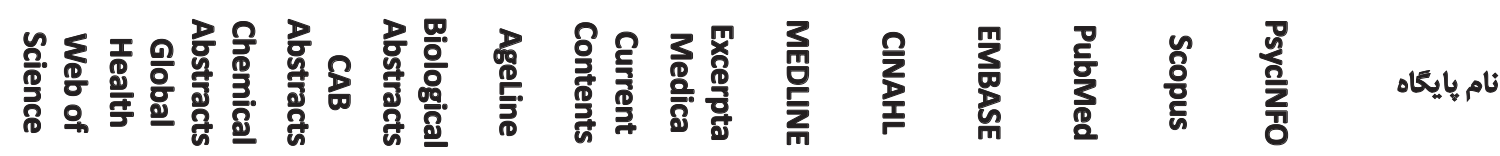

\begin{tabular}{|c|c|c|c|c|c|c|c|c|c|c|c|c|c|c|}
\hline$r A / I r$ & . & r. & $r+/ r i$ & 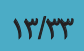 & $Q \cdot M^{*}$ & $r \Delta / \pi r$ & $M / \Delta r$ & $\Delta H / F T$ & $M T / \cdot r$ & $r q / v q$ & PV/TV & $r q / \cdot r$ & • & PsycINFO \\
\hline QNIA & . & IV/AQ & $\mid$ W9| & IT/AF & $\Gamma / M$ & $\Delta \cdot / \% \Delta$ & $\mu F / / F$ & LIT/M & ples & $V I / \& A$ & $V Q / T r$ & . & $r q / \cdot v$ & Scopus \\
\hline$\Delta V / \& \Delta$ & . & IV/rT & WVA & $I r / v e$ & TT/MT & Q. & rT/Fr & $\Delta T / Y \Delta$ & rNM & $E Q / \Delta E$ & ${ }^{\circ}$ & va/me & $\mathrm{FV} / \mathrm{TV}$ & PubMed \\
\hline g. & . & $r \cdot 19 q$ & $r+/ A Y$ & 181.9 & $\Gamma \Delta / r " \Delta$ & Ar/SA & $8+148$ & 98189 & $\mathbb{R} / \wedge q$ & - & $g q / \Delta g$ & $V D / 8 \mathrm{~A}$ & $r e / v q$ & EMBASE \\
\hline TNVA & - & WAI & WQS & $9 / 9$ & $\Delta \Delta$ & $\mathrm{HY} / \mathrm{TA}$ & WTa & Tr/Fe & . & $r V / A q$ & rNMY & FI/Fg & $\mathrm{FT} / . \mathrm{T}$ & CINAHL \\
\hline gepry & - & $r \Delta / A$. & TA/NA & IV/er & Pr/Fe & selse & $M T / N F$ & . & er/pe & sqles & $\Delta T / T \Delta$ & $\Delta T / M$ & AH/RT & MEDLINE \\
\hline$r \cdot / P$ & . & WTr & WNA & $19 / 97$ & W/F & T & . & $m / N F$ & WTI & 8.118 & Pr/er & $r F / 1 P$ & TH/AT & Excerpta Medica \\
\hline$A Y / \cdot 9$ & . & $\mathrm{rV} / \mathrm{II}$ & TQ & $18 / \pi q$ & RT/PE & - & $M r / M r$ & $98 / 89$ & Pe/rs & $\Delta T / \& A$ & Q. & $\Delta . / P \Delta$ & $P \Delta / \pi$ & Current Contents \\
\hline$r q / \cdot r$ & . & $|Q / \Delta|$ & $1 E / T$ & NEY & . & $r t / R T$ & ז'T/F & Pr/ge & $\Delta \Delta$ & 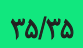 & PT/NT & $r+/ 11$ & $\Delta . / M^{\circ}$ & Ageline \\
\hline $19 / 11$ & . & PIIFE & $r \Delta / A$. & . & NET & $18 / \pi q$ & $19 / 97$ & IV/ee & $9 / \div 9$ & 191.9 & IT/NE & IY/AF & $1 \pi / m$ & $\begin{array}{l}\text { Biological } \\
\text { Abstracts }\end{array}$ \\
\hline MT/MA & - & $\pi / M$ & - & $r \Delta / A$. & $19 / T$ & YQ & WNA & $r \Delta / V \Delta$ & WQ & $r \cdot / A V$ & INVD & W91 & $r \cdot /{ }^{\prime}$ & CAB Abstracts \\
\hline$M e / M r$ & . & . & 侻 & piles & $|\Delta / \Delta|$ & $r V / M$ & $\mathbb{N} / \mathrm{T}$ & $r \Delta / A$. & WAI & $r \cdot 19 q$ & IV/er & IV/0Q & r. & Chemical Abstracts \\
\hline . & - & - & - & . & . & - & . & . & - & - & - & . & - & Global Health \\
\hline . & . & $M e / M P$ & TH/TA & $19 / 11$ & $r q / \cdot r$ & $A r / \circ 9$ & $p=1 P V$ & ST/PV & TNVQ & 8. & $\Delta V / \& \Delta$ & QNIN & $P A / I T$ & Web of Science \\
\hline
\end{tabular}

$$
\begin{aligned}
& 1+-1 . \cdot \\
& 8+-1 . \\
& r+-8 . \\
& r+-r \cdot \\
& 1-r+
\end{aligned}
$$

il

اين در حالى است كه نتايج اين مطالعه نشان داد تنها

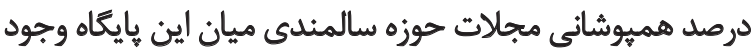

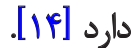

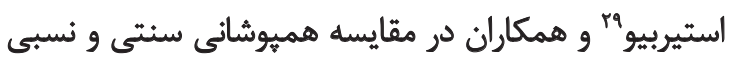

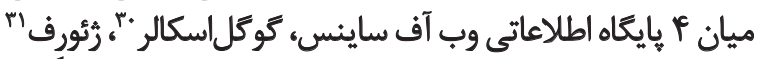

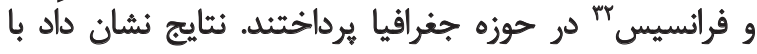

\section{9. Ştirbu}

30. Google Scholar

31. GEOREF

32. FRANCIS
ميزان هميوشانى دو يايخًاه در حوزه سالمندى بيشتر از حوزه بيمارىهاى غدد درونريز بوده است.

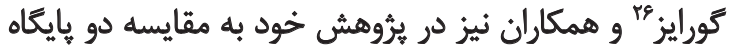

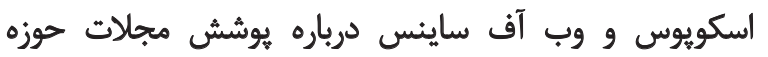

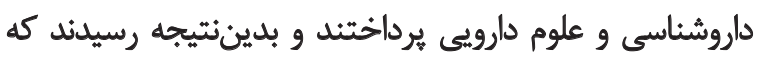

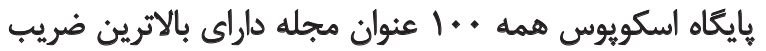

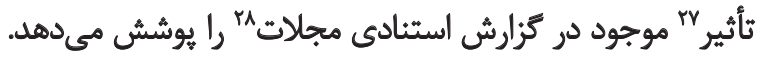

28. Journal Citation Report (JCR) 
جدول هـ. وضعيت تأمين كنيدكان متن كامل مثالات مجلات حوزه سالمئدى.

\begin{tabular}{|c|c|c|}
\hline درصد & ت تعداد مجلات & نام تأمين كتنده متن كامل \\
\hline QNVD & 1.9 & EBSCOhost \\
\hline$P \varphi / .8$ & $\mathrm{rA}$ & Swets \\
\hline HNFI & 91 & Copyright Clearance Center \\
\hline 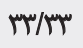 & $\Delta 9$ & Scholars Portal \\
\hline $\mathrm{r} / \mathrm{u} / \mathrm{u}$ & te & OCLC \\
\hline$M \in / \wedge \Delta$ & m & OhioLINK \\
\hline War & r & ProQuest \\
\hline$W \cdot V$ & rit & Health Communication Network \\
\hline $1 \otimes / T \Delta$ & rV & Ovid \\
\hline$I T / R T$ & $\pi$ & Directory of Open Access Journals (DOAJ) \\
\hline$I K / A T$ & rr & Gale \\
\hline $11 / 79$ & r. & Elsevier \\
\hline$\sqrt{ } / \%$ & IV & Springer \\
\hline$\checkmark / \varepsilon$. & IV & Taylor \& Francis \\
\hline$V / M F$ & r & National Library of Medicine \\
\hline$g / W$ & ir & LexisNexis \\
\hline$\Delta / \bullet \wedge$ & 9 & SAGE Publications \\
\hline$r / r \Delta$ & f & BioMedCentral \\
\hline$r / r \Delta$ & f & Cambridge University Press \\
\hline T/RA & $f$ & Lippincott Williams \& Wilkins \\
\hline$r / r \Delta$ & f & Oxford University Press \\
\hline$t / T \Delta$ & r & Wiley-Blackwell \\
\hline M & r & Emerald \\
\hline
\end{tabular}

닌

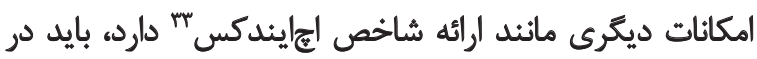

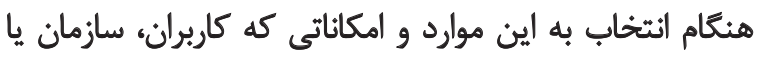
كتابخانه بدان نياز دارند نيز توجه كرابن

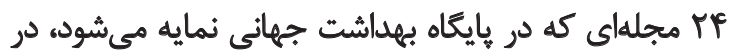

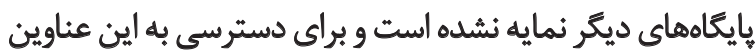

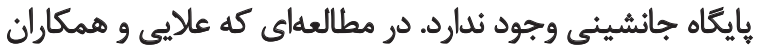

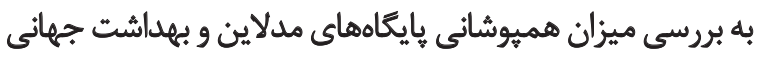

33. H-index

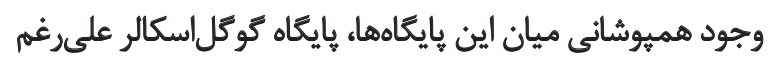

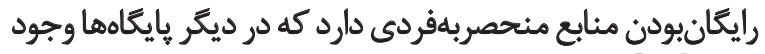
ندارد [10]

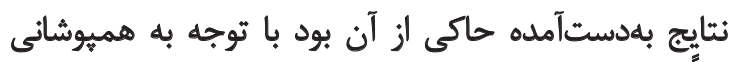

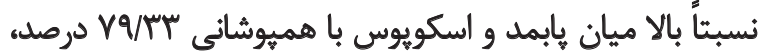

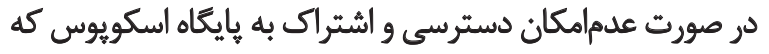

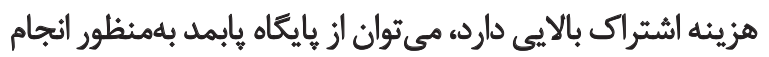

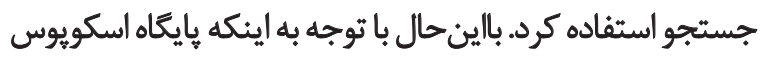




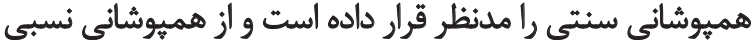

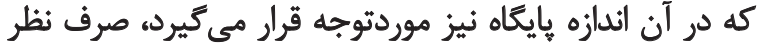

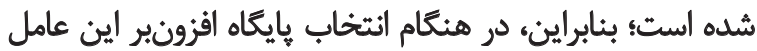

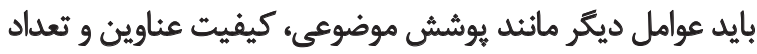
سال هاى زيريوشش را نيز مدنظر قرار داد.

$$
\text { تشكر و قدوداني }
$$

از مركز تحقيقات سالمندى دانشكاه علوم بهزيستى و

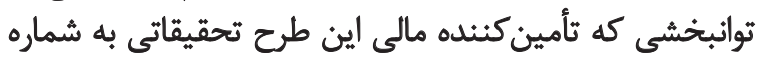

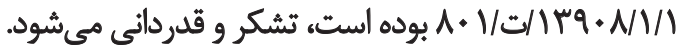

درباره يوشش اطلاعات حوزه بهداشت عمومى برداختهاند، نتايج

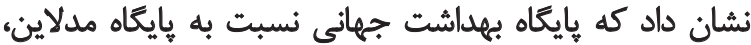

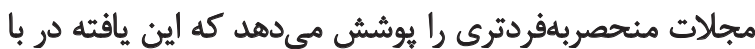

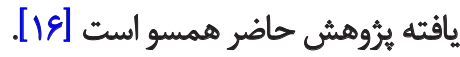

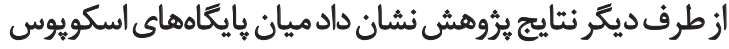

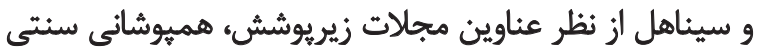

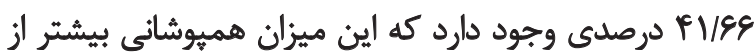

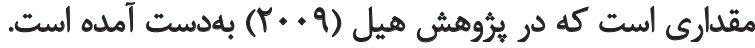

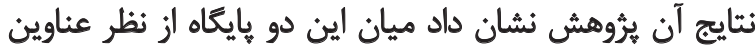

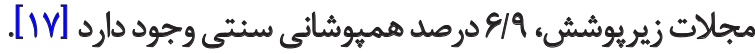
در مطالعهاى كه توسط فرجيهلو و مكىزاده كه درباره هزينه-

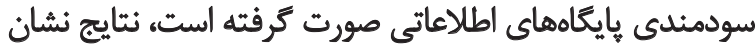

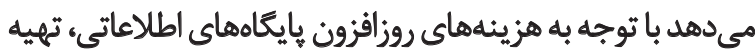

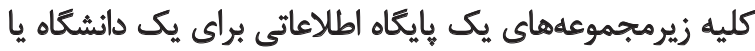

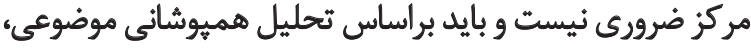

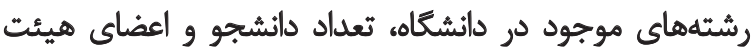

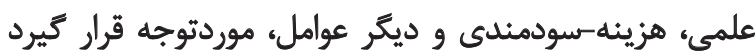

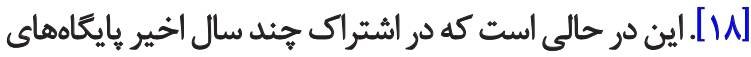
اطلاعاتي كشور كه بهصورت متمركز و شراكتي انجام كرفتئه اين

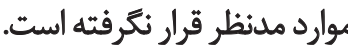

\section{نتيجديرى نهايي}

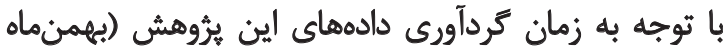

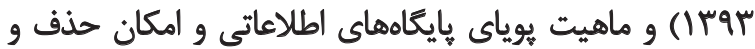

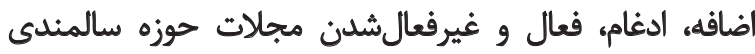

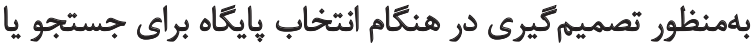

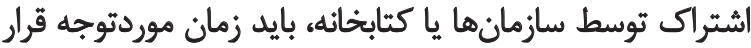

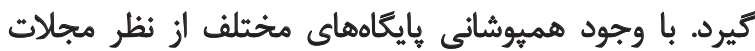

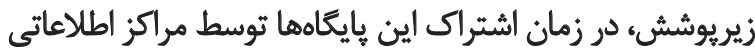

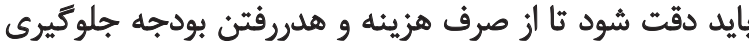

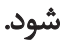

ازظرفديكر، وجود هميوشانى در يايكاههاى مختلف باعث

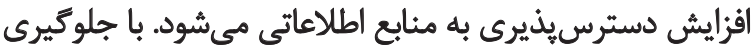

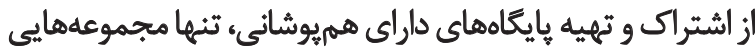

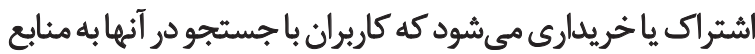

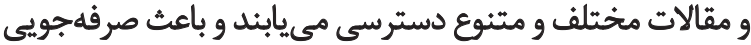

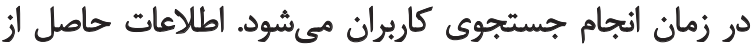

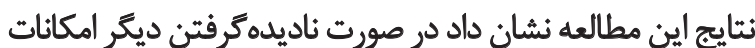

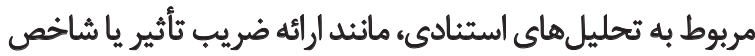

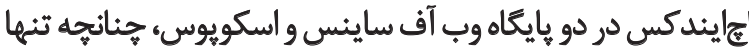

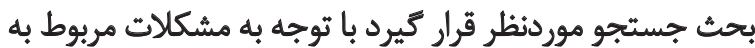

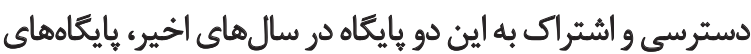

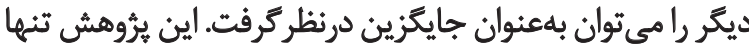




\section{References}

[1] Tanjani PT, Motlagh ME, Nazar MM, Najafi F. The health status of the elderly population of Iran in 2012. Archives of Gerontology and Geriatrics. 2015; 60(2):281-87.

[2] Mirzaie M, Shams M. [Demographic of elderly in Iran Based on 1956-2006 headcounts (Persian)]. Iranian Journal of Ageing. 2007; 2(3):326-31.

[3] Hatami H, Razavi SM, Ardebili HE, Majlesi F. [Textbook of public health (Persian)]. Tehran: Arjmand Press; 2013.

[4] Blessinger K, Olle M. Content analysis of the leading general academic databases. Library Collections, Acquisitions, and Technical Services. 2004; 28(3):335-46.

[5] Gavel Y, Iselid L. Web of Science and Scopus: a journal title overlap study. Online Information Review. 2008; 32(1):8-21.

[6] Nooshinfard F, Emami Z. Traditional and relative overlap of "PubMed" and "Web of Science" databases in the field of endocrine diseases. Journal of Information System and Services. 2010; 3(1):89-101.

[7] Davarpanah MR. [Scientific information seeking in printed and electronic resources (Persian)]. $2^{\text {nd }}$ ed. Tehran: Chapar Press; 2007.

[8] Ghazimirsaeed SJ, Pouramini Z. [Overlap articles of respiratory system in databases Scopus and Web of Science: brief report (Persian)]. Tehran University Medical Journal. 2015; 72(12):854-59.

[9] Hood WW, Wilson CS. Overlap in bibliographic databases. Journal of the American Society for Information Science and Technology. 2003; 54(12):1091-103.

[10] Jacsó P. Analysis of the Ulrich's serials analysis system from the perspective of journal coverage by academic databases. Online Information Review. 2012; 36(2):307-19.

[11] Fattahi R, Mansourian Y, Arastoopour S. [Serials Management Handbook (Persian)]. $2^{\text {nd }}$ ed. Tehran: Dabizesh; 2010.

[12] Alibeiq MR, Jamshidi Orak R, Asghari Heineh Abad L. [A Survey on traditional overlap, relative overlap and synthetic degrees of freedom between PubMed and Scopus in the cardiovascular disease field (Persian)]. Journal of Health Information Management. 2011; 8(3):345-53.

[13] Diodato VP. [Dictionary of Bibliometrics (Gh. Heydari, R. Khademi, Persian trans)]. Tehran: Ketabdar Press; 2011.

[14] Gorraiz J, Schloegl C. A bibliometric analysis of pharmacology and pharmacy journals: Scopus versus Web of Science. Journal of Information Science. 2008; 34(5):715-25.

[15] Ştirbu S, Thirion P, Schmitz S, Haesbroeck G, Greco N. The utility of Google Scholar when searching geographical literature: comparison with three commercial bibliographic databases. Journal of Academic Librarianship. 2015; 41(3):322-29.

[16] Aalai E, Gleghorn C, Webb A, Glover SW. Accessing public health information: a preliminary comparison of CABI's Global Health database and Medline. Health Information \& Libraries Journal. 2009; 26(1):56-62.

[17] Hill B. Comparison of journal title coverage between CINAHL and Scopus. Journal of Medical Library Association. 2009; 97(4):313-14.
[18] Makkizadeh F, Faraj Pahloo A. A survey of costs-benefit of databases in Yazd university in 2009. Journal of Academic Librarianship. 2010; 44(54):59-82. 